\title{
Dissipative Floquet dynamical quantum phase transition
}

\author{
J. Naji, ${ }^{1, *}$ Masoud Jafari $\odot,{ }^{2, \dagger}$ R. Jafari $\odot,{ }^{3,4,5, \dagger}$ and Alireza Akbari $\odot^{6,7, \S}$ \\ ${ }^{1}$ Department of Physics, Faculty of Science, Ilam University, Ilam, Iran \\ ${ }^{2}$ Department of Computer Engineering, Iran University of Science and Technology, Tehran, Iran \\ ${ }^{3}$ Department of Physics, Institute for Advanced Studies in Basic Sciences (IASBS), Zanjan 45137-66731, Iran \\ ${ }^{4}$ Department of Physics, University of Gothenburg, SE-412 96 Gothenburg, Sweden \\ ${ }^{5}$ Beijing Computational Science Research Center, Beijing 100094, China \\ ${ }^{6}$ Max Planck Institute for the Chemical Physics of Solids, D-01187 Dresden, Germany \\ ${ }^{7}$ Max Planck POSTECH Center for Complex Phase Materials, and Department of Physics, POSTECH, Pohang, Gyeongbuk 790-784, Korea
}

(Received 26 October 2021; accepted 14 February 2022; published 28 February 2022)

\begin{abstract}
Non-Hermitian Hamiltonians provide a simple picture for inspecting dissipative systems with natural or induced gain and loss. We investigate the Floquet dynamical phase transition in the dissipative periodically time-driven $X Y$ and extended $X Y$ models, where the imaginary terms represent the physical gain and loss during the interacting processes with the environment. The time-independent effective Floquet non-Hermitian Hamiltonians disclose three regions by analyzing the non-Hermitian gap: pure real gap (real eigenvalues), pure imaginary gap, and complex gap. We show that each region of the system can be distinguished by the complex geometrical nonadiabatic phase. We have discovered that in the presence of dissipation, the Floquet dynamical phase transitions (FDPTs) still exist in the region where the time-independent effective Floquet non-Hermitian Hamiltonians reveal real eigenvalues. Opposed to expectations based on earlier works on quenched systems, our findings show that the existence of the non-Hermitian topological phase is not an essential condition for dissipative FDPTs (DFDPTs). We also demonstrate the range of driven frequency, over which the DFDPTs occur, narrows down by increasing the dissipation coupling and shrinks to a single point at the critical value of dissipation. Moreover, quantization and jumps of the dynamical geometric phase reveals the topological characteristic feature of DFDPTs in the real gap region where confined to exceptional points.
\end{abstract}

DOI: 10.1103/PhysRevA.105.022220

\section{INTRODUCTION}

Non-Hermitian Hamiltonians have recently attracted a lot of attention in the physics community across a wide range of fields, owing to their experimental feasibility [1-18] and theoretical richness [19-28]. Quantum systems driven by non-Hermitian Hamiltonians display various fascinating physical phenomena comparing to those governed by Hermitian Hamiltonians. In cold-atom experiments, non-Hermitian Hamiltonians appear due to spontaneous decay [21,29-32]. Furthermore, various non-Hermitian Hamiltonians have been utilized to treat various physical problems that might need to consider the interaction between the environment and the system, such as free-electron lasers [33], topological lasers

\footnotetext{
*j.naji@ilam.ac.ir

†masoud_jafari@comp.iust.ac.ir

*jafari@iasbs.ac.ir, raadmehr.jafari@gmail.com

§akbari@cpfs.mpg.de
}

Published by the American Physical Society under the terms of the Creative Commons Attribution 4.0 International license. Further distribution of this work must maintain attribution to the author(s) and the published article's title, journal citation, and DOI. Open access publication funded by the Max Planck Society.
[34-36], electric circuit [37-39], transverse mode propagation in optical resonators [40], multiphoton ionization [41], many resonance phenomena [42], nitrogen-vacancy center in diamond $[43,44]$, with applications on high performance sensors $[6,10,45,46]$, and unidirectional transport devices $[13,47]$.

Theoretically, non-Hermitian Hamiltonians trigger many novel physical phenomena, such as non-Hermitian skin effect $[48,49]$, real eigenvalues with parity-time (PT) symmetry [50], new topological properties corresponding to exceptional points (EPs) [51-55], disorder induced self-energy in the effective Hamiltonian [56-60], and dynamical and topological properties $[4,15,54,61,62]$. Finding the dynamical signatures of these nonequilibrium topological matter has become a fascinating area for more experimental and theoretical research. In recent works, several dynamical probes to the topological invariants of non-Hermitian phases in one and two dimensions have been introduced, such as the nonHermitian extension of dynamical winding numbers [63-68] and mean chiral displacements $[69,70]$. Further, the dynamical quantum phase transitions (DQPTs) [71-99] following a quench across the EPs of a non-Hermitian lattice model is studied in Refs. [63,64]. It has been shown that DQPTs appear for a quench from a trivial to a non-Hermitian topological phase [64]. This discovery indicates an underlying relationship between non-Hermitian topological phases and DQPTs. 
To the best of our knowledge, the Floquet dynamical phase transition in the systems with gain and loss, and therefore subject to nonunitary evolution, have not been addressed in prior publications and can provide a number of new insights into the subject. This paper is devoted to the research on the Floquet dynamical phase transition [100-105] in the periodically time-driven $X Y$ and extended $X Y$ spin models in the presence of dissipation. The non-Hermitian terms (imaginary terms) represent dissipation - the physical gain and losswhen the chain interacts with the environment. Our main purpose is to study the effects of non-Hermitian terms on the FDPTs time and the range of driven frequency over which the DFDPTs occur. First, we probe the phase diagram of the time-independent effective Floquet non-Hermitian Hamiltonians by analyzing the energy gap of the systems analytically. We show that the phase diagram of the system divided into three regions with pure real gap where confined to exceptional points, pure imaginary gap, and complex gap. We have found that, the region with real energy gap, where the DFDPTs occur, is topologically nontrivial in the time-independent effective Floquet non-Hermitian $X Y$ Hamiltonian. While the real gap region in the time-independent effective Floquet nonHermitian extended $X Y$ Hamiltonian is topologically trivial. In the other words, different from results obtained for the quenched case [64], existence of the non-Hermitian topologically nontrivial phase is not a necessary condition for appearance of the DFDPTs. We have also shown that, the DFDPTs driven frequency range narrows down by increasing the dissipation coupling and shrinks to a single point at critical value of dissipation. We have found that adding the dissipation (imaginary term) to the Hermitian Hamiltonians affects those bounds of the driven frequency range which correspond to the critical (gap closing) points of the time-independent effective Floquet Hermitian Hamiltonians.

\section{DYNAMICAL PHASE TRANSITION}

The notion of a DQPT borrowed from the analogy between the partition function of an equilibrium system $Z(\beta)=\operatorname{Tr}\left[e^{-\beta \mathcal{H}}\right]$ and the boundary quantum partition function $Z(z)=\left\langle\psi_{0}\left|e^{-z \mathcal{H}}\right| \psi_{0}\right\rangle$ with $\left|\psi_{0}\right\rangle$ a boundary state and $z \in \mathbb{C}$. When $z=i t$, the boundary quantum partition function corresponds to a Loschmidt amplitude (LA), $\mathcal{L}(t)=$ $\left\langle\psi_{0}\left|e^{-i \mathcal{H} t}\right| \psi_{0}\right\rangle$, expressing the overlap between the initial state $\left|\psi_{0}\right\rangle$ and the time-evolved one $\left|\psi_{0}(t)\right\rangle$ [71-95,102-105]. It has been argued that, like the thermal free energy, a dynamical free energy might well be defined as [71]

$$
g(t)=-\frac{1}{N} \lim _{N \rightarrow \infty} \ln |\mathcal{L}(t)|^{2} .
$$

Here the real time $t$ plays the role of the control parameter and $N$ is the size of the system. DQPTs are signaled by nonanalytical behavior of dynamical free energy $g(t)$ as a function of time, evincing in characteristic cusps in $g(t)$ or one of its time derivatives [71-95,102-105]. These cusps are followed by zeros of Loschmidt amplitude $\mathcal{L}(t)$, known in statistical physics as Fisher zeros of the partition function [75,92]. Furthermore, analogous to order parameters at equilibrium quantum phase transition, a dynamical topological order parameter (DTOP) is proposed to capture DQPTs [74].
The DTOP is quantized and its unit magnitude jumps at the DQPT time reveals the topological characteristic feature of DQPT $[74,81]$. This dynamical topological order parameter is extracted from the gauge-invariant Pancharatnam geometric phase associated with the Loschmidt amplitude [74].

The dynamical topological order parameter is defined as [74]

$$
v_{D}(t)=\frac{1}{2 \pi} \int_{0}^{\pi} \frac{\partial \phi^{G}(k, t)}{\partial k} d k,
$$

where the geometric phase $\phi^{G}(k, t)$ is gained from the total phase $\phi(k, t)$ by subtracting the dynamical phase $\phi^{D}(k, t)$ :

$$
\phi^{G}(k, t)=\phi(k, t)-\phi^{D}(k, t) .
$$

The total phase $\phi(k, t)$ is the phase factor of LA in its polar coordinates representation, i.e., $\mathcal{L}_{k}(t)=\left|\mathcal{L}_{k}(t)\right| e^{i \phi(k, t)}$, results $\phi(k, t)=-i \ln \left[\mathcal{L}_{k}(t) /\left|\mathcal{L}_{k}(t)\right|\right]$, and

$$
\begin{aligned}
\phi^{D}(k, t)= & -\int_{0}^{t} d t^{\prime} \frac{\left\langle\psi_{-}\left(k, t^{\prime}\right)\left|\mathcal{H}_{k}\left(t^{\prime}\right)\right| \psi_{-}\left(k, t^{\prime}\right)\right\rangle}{\left\langle\psi_{-}\left(k, t^{\prime}\right) \mid \psi_{-}\left(k, t^{\prime}\right)\right\rangle} \\
& +\frac{i}{2} \ln \left[\frac{\left\langle\psi_{-}(k, t) \mid \psi_{-}(k, t)\right\rangle}{\left\langle\psi_{-}(k, 0) \mid \psi_{-}(k, 0)\right\rangle}\right] .
\end{aligned}
$$

In following, to examine aspects of dissipative in quantum Floquet systems, we search for dissipative Floquet DPTs in proposed non-Hermitian periodically time-driven Hamiltonians.

\section{DISSIPATIVE PERIODICALLY TIME-DRIVEN $X Y$ MODEL AND EXACT SOLUTION}

In this section we study the phase diagram, topological properties, and FDPTs of the dissipative periodically timedriven $X Y$ model. We show that the region in which DFDPTs occur is confined to exceptional points and is topologically nontrivial and the time-independent effective Floquet nonHermitian Hamiltonian has real eigenvalues.

\section{A. Exact solution}

The Hamiltonian of the $N$-site dissipative periodically time-driven $X Y$ spin model is given as

$$
\begin{aligned}
\mathcal{H}(t)=\sum_{n} & {\left[[J-\gamma \cos (\omega t)] S_{n}^{x} S_{n+1}^{x}+[J+\gamma \cos (\omega t)] S_{n}^{y} S_{n+1}^{y}\right.} \\
& -\gamma \sin (\omega t)\left(S_{n}^{x} S_{n+1}^{y}+S_{n}^{y} S_{n+1}^{x}\right)+h S_{n}^{z} \\
& \left.-i\left(\Gamma_{u} S_{n}^{+} S_{n}^{-}+\Gamma_{d} S_{n}^{-} S_{n}^{+}\right)\right]
\end{aligned}
$$

where $S_{n}^{\alpha=\{x, y, z\}}=\sigma^{\alpha} / 2$, and $\sigma^{\alpha}$ are Pauli matrices. Furthermore, $S_{n}^{ \pm}=\sigma^{ \pm} / 2=\left(\sigma^{x} \pm i \sigma^{y}\right) / 2$ are the spin raising and lowering operators which correspond to the gain $\Gamma_{u}<0$ $\left(\Gamma_{d}<0\right)$ or loss $\Gamma_{u}>0\left(\Gamma_{d}>0\right)$ of the spin-up state $|\uparrow\rangle$ (spin-down state $|\downarrow\rangle$ ) during the interacting processes with the environment with the rate of $\Gamma_{u}\left(\Gamma_{d}\right)$, and $\omega$ is the driving frequency. The system can be reduced to the Floquet Hermitian $X Y$ model when $\Gamma_{u}=\Gamma_{d}=0$ [105]. The term "dissipative" refers to the system's tunneling effects to its own continuum, which is common in quantum optics and nuclear physics when using the Feshbach projection method on intrinsic states. 
The Hamiltonian, Eq. (1), can be mapped to the free spinless fermion model with complex chemical potential [106] by Jordan-Wigner transformation [107-110] (see Appendix A):

$$
\begin{aligned}
\mathcal{H}(t)= & \sum_{n=1}^{N}\left[\left(\frac{J}{2} c_{n}^{\dagger} c_{n+1}-\frac{\gamma}{2} e^{-i \omega t} c_{n}^{\dagger} c_{n+1}^{\dagger}+\text { H.c. }\right)\right. \\
& \left.+\left(h-i \Gamma_{-}\right) c_{n}^{\dagger} c_{n}-i \Gamma_{+}\right],
\end{aligned}
$$

where $\Gamma_{ \pm}=\Gamma_{u} \pm \Gamma_{d}$, and $c_{n}^{\dagger}\left(c_{n}\right)$ are the spinless fermion creation (annihilation) operators, respectively. Thanks to the Fourier transform, the Hamiltonian $\mathcal{H}(t)$ in Eq. (5) can be written as the sum of $N / 2$ noninteracting terms,

$$
\mathcal{H}(t)=\sum_{k>0} \mathcal{H}_{k}(t)
$$

where $\mathcal{H}_{k}(t)=C^{\dagger} \uplus_{k}(t) C-i \Gamma_{+} \mathbb{1}$ with $C^{\dagger}=\left(c_{k}^{\dagger}, c_{-k}\right)$, and

$$
\mathbb{H}_{k}(t)=\left(\begin{array}{cc}
h_{z}(k) & i h_{x y}(k) e^{-i \omega t} \\
-i h_{x y}(k) e^{i \omega t} & -h_{z}(k)
\end{array}\right) .
$$

The parameters $h_{x y}(k)$ and $h_{z}(k)$ are given as $h_{x y}(k)=$ $\gamma \sin (k)$, and $h_{z}(k)=J \cos (k)+h-i \Gamma_{-}$. Using the timedependent Schrödinger equation $i \frac{d}{d t}\left|\psi_{k}^{ \pm}(t)\right\rangle=\leftrightarrow_{k}(t)\left|\psi_{k}^{ \pm}(t)\right\rangle$ in the rotating frame given by the nonunitary transformation $U(t)=U_{R}(t) U_{D}(t)$, with $U_{R}(t)=\exp \left[i \omega\left(\mathbb{1}-\sigma^{z}\right) t / 2\right]$, and $U_{D}(t)=e^{-\Gamma_{+} t} \mathbb{1}$, the time-dependent Hamiltonian is transformed to the time-independent effective Floquet nonHermitian form (see Appendix A):

$$
H_{F}(k)=-h_{x y}(k) \sigma_{y}+\left(h_{z}(k)-\frac{\omega}{2}\right) \sigma_{z}+\frac{\omega}{2} \mathbb{1} .
$$

Then the time-evolved $\left|\psi_{k}(t)\right\rangle$ of the quasispin Hamiltonian $H_{k}(t)$ is given by

$$
\left|\psi_{k}(t)\right\rangle=U(t) e^{-i H_{F}(k) t}\left|\varphi_{k}\right\rangle,
$$

where $\left|\varphi_{k}\right\rangle$ is the initial state of the system at $t=0$. Due to the decoupling of different momentum sectors, the initial and time-evolved ground states of the original Hamiltonian exhibit a factorization property that is expressed by

$$
\begin{aligned}
& |\psi(t)\rangle=\prod_{k}\left|\psi_{k}(t)\right\rangle=\prod_{k} U(t) e^{-i H_{F}(k) t}\left|\varphi_{k}\right\rangle, \\
& |\psi(t=0)\rangle=\prod_{k}\left|\varphi_{k}\right\rangle .
\end{aligned}
$$

We consider that at $t=0$ the system prepared at $|\psi(0)\rangle=$ $\left|\varphi_{k}\right\rangle=|\downarrow\rangle$, i.e., $c_{1}(t=0)=0$ and $c_{2}(t=0)=1$, where $c_{1}$ and $c_{2}$ are probability amplitudes of $|\psi(0)\rangle$ at up $(|\uparrow\rangle)$ and down ( $|\downarrow\rangle)$ states, respectively. Then according to Eq. (9) the un-normalized time-evolving state $|\psi(k, t)\rangle$ of the Hamiltonian $\mathcal{H}_{k}(t)$ is given by

$$
|\psi(t)\rangle=\prod_{k}|\psi(k, t)\rangle,
$$

with

$$
|\psi(k, t)\rangle=\left[e^{-\Gamma_{+} t}\left(\frac{h_{x y}(k)}{\Lambda} \sin (\Lambda t)\right)|\uparrow\rangle\right.
$$

$$
\left.+e^{-\Gamma_{+} t} e^{i \omega t}\left(\cos (\Lambda t)+i \frac{2 h_{z}(k)-\omega}{2 \Lambda} \sin (\Lambda t)\right)|\downarrow\rangle\right],
$$

and $\Lambda=\sqrt{h_{x y}^{2}(k)+\left[h_{z}(k)-\frac{\omega}{2}\right]^{2}}$.

The time-independent effective Floquet non-Hermitian Hamiltonian in Eq. (7), possesses the sublattice symmetry $U_{s} H(k) U_{s}^{-1}=-H(k)$ with $\mathcal{S}=\sigma_{x}$, and generalized particlehole symmetry $U_{p} H^{\top}(k) U_{p}^{-1}=-H(-k)$, as well as, the time-reversal symmetry $U_{T} H^{\top}(k) U_{T}^{-1}=H(-k)$ with $U_{p}=$ $\sigma_{x}$ and $U_{T}=\mathbb{1}$. Here $H^{\top}(k)$ is transposed of $H^{\top}(k)$. Consequently, the symmetry class of the non-Hermitian time-independent Hamiltonian in Eq. (7) belongs to BDI in the periodic table of non-Hermitian topological phases [55]. Moreover, $H_{F}(k)$ encompasses the inversion symmetry $U_{I} H(k) U_{I}^{-1}=H(-k)$ with $U_{I}=\sigma_{z}$, which manifests the correspondence between the bulk topological invariant and the number of Majorana edge modes under the open boundary condition $[55,106]$.

The complex energy spectrum of $H_{F}(k)$ is given as

$$
\varepsilon_{k}^{ \pm}=\frac{\omega}{2} \pm \sqrt{h_{x y}^{2}(k)+\left[h_{z}(k)-\frac{\omega}{2}\right]^{2}},
$$

and becomes gapless if

$$
\begin{array}{r}
2 \Gamma_{-}\left[J \cos (k)+h-\frac{\omega}{2}\right]=0, \\
{\left[J \cos (k)+h-\frac{\omega}{2}\right]^{2}+[\gamma \sin (k)]^{2}-\Gamma_{-}^{2}=0 .}
\end{array}
$$

By solving the above equations, we can get

$$
\begin{aligned}
& k^{*}=\arccos \left(\frac{\omega-2 h}{2 J}\right), \\
& \frac{\Gamma_{-}^{2}}{\gamma^{2}}+\frac{(\omega-2 h)^{2}}{4 J^{2}}=1 .
\end{aligned}
$$

Equation (13) implies a limitation $\omega-2 h< \pm 2 J$ and Eq. (14) depicts an elliptical exceptional ring. Therefore, the system can be separated into three regions as shown in Fig. 1. In the region (I), inside the exceptional ring, the energy gap (eigenvalues) $\Delta=\left|\varepsilon_{K}^{+}-\varepsilon_{k}^{-}\right|$is purely real, i.e., $\operatorname{Im}[\Delta]=0$ and $\operatorname{Re}[\Delta]>0(\operatorname{Im}[\mathbb{C}]$ and $\operatorname{Re}[\mathbb{C}]$ represent the imaginary and real part of complex number $\mathbb{C}$, respectively). In this region $k^{*}=\arccos [(\omega-2 h) /(2 J)]$ and $\Delta=\sqrt{\gamma^{2}\left[1-(\omega-2 h)^{2} /\left(4 J^{2}\right)\right]-\Gamma_{-}^{2}}$. In region (II) the gap is pure imaginary, i.e., $\operatorname{Im}[\Delta] \neq 0$ and $\operatorname{Re}[\Delta]=0$. In this region we still have $k^{*}=\arccos [(\omega-2 h) /(2 J)]$ but the nonHermitian strength $\Gamma_{-}$is large enough to be dominant, then $\Delta=i \sqrt{\Gamma_{-}^{2}-\gamma^{2}\left[1-(\omega-2 h)^{2} /\left(4 J^{2}\right)\right]}$. Region (III) ( $\mid \omega-$ $2 h \mid>2 J)$ is characterized by the complex gap. In other words, in region (III) both real and imaginary parts of the gap are nonzero.

\section{B. Complex geometrical nonadiabatic phase}

In this section we study the geometric phase of the model to show how the geometric phase can detect the three regions 


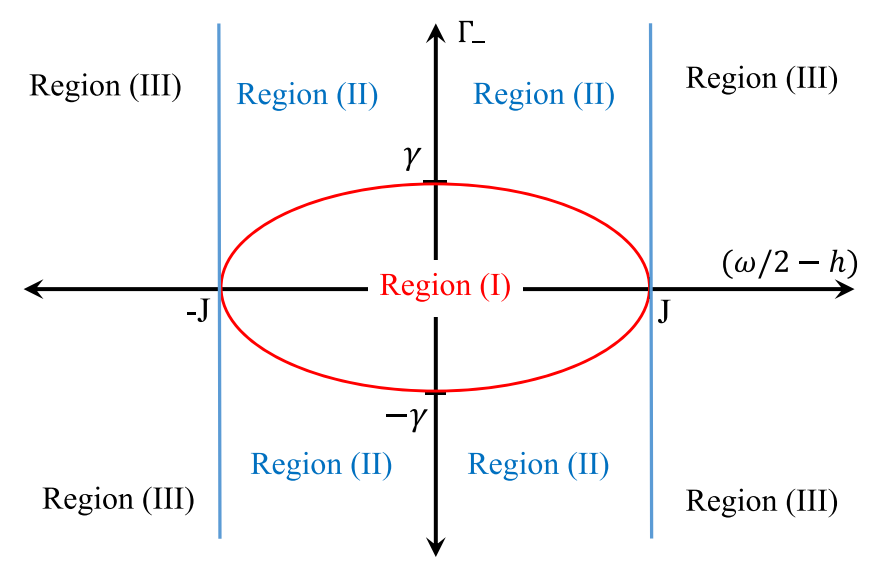

FIG. 1. The phase diagram of the time-independent effective Floquet non-Hermitian $X Y$ Hamiltonian. The red line denotes the exceptional ring, which corresponds to the elliptic equation [Eq. (13)]. In region (I), the system is in the ferromagnetic phase with a pure real energy gap. In region (II) the energy gap of the non-Hermitian Hamiltonian is a pure imaginary. In region (III) it is in the paramagnetic phase with a complex non-Hermitian gap.

in the time-independent effective Floquet non-Hermitian $X Y$ Hamiltonian mentioned in the previous section. For the nonadiabatic evolutions we use the Lewis-Riensenfeld invariant theory [111] which generalized to non-Hermitian systems $[112,113]$. According to Lewis-Riensenfeld theory the nonHermitian invariant $I(t)$ associated with the Hamiltonian $\mathrm{H}_{k}(t)$, Eq. (6), can be expressed as linear combinations of Pauli matrices, i.e.,

$$
I(t)=r_{1} S^{+}+r_{2}(t) S^{-}+r_{3}(t) S^{z},
$$

where $r_{m=\{1,2,3\}}(t)$ are three time-dependent complex parameters and $I(t)$ satisfies the Liouville-von Neumann equation,

$$
\frac{d}{d t} I(t)=\frac{\partial}{\partial t} I(t)-i\left[I(t), \mathbb{H}_{k}(t)\right] .
$$

The substitution of expressions of $I(t)$ and $\sharp_{k}(t)$ in Eq. (15) leads to the system of coupled differential equations. By solving the coupled differential equations, which satisfies the cyclicity of $I(t+T)=I(t)$ with $T=2 \pi / \omega$ results,

$$
I(t)=\left(\begin{array}{cc}
\cos (\alpha) & \sin (\alpha) e^{-i \omega t} \\
\sin (\alpha) e^{i \omega t} & -\cos (\alpha)
\end{array}\right),
$$

where $\cos (\alpha)=\frac{2 h_{z}(k)-\omega}{\sqrt{4 h_{x y}^{2}(k)+\left[2 h_{z}(k)-\omega\right]^{2}}}$.

The complex geometrical nonadiabatic phase for a cyclic evolution $T=2 \pi / \omega$ is defined by $[112,113]$

$$
\beta(t)=i \int_{0}^{T}\left\langle\Phi_{-}\left|\frac{\partial}{\partial t}\right| \Psi_{-}\right\rangle d t
$$

where $\left|\Psi_{-}\right\rangle$and $\left|\Phi_{-}\right\rangle$are the instantaneous eigenstates of $I(t)$ and $I(t)^{\dagger}$ (see Appendix B). The complex geometrical nonadiabatic phase for the periodically time-driven dissipative Floquet $X Y$ model is obtained as

$$
\beta=\pi[1-\cos (\alpha)]=\pi\left[1-\frac{2 h_{z}(k)-\omega}{\sqrt{4 h_{x y}^{2}(k)+\left[2 h_{z}(k)-\omega\right]^{2}}}\right],
$$

which is a generalization of the complex solid angle in complex parameter space [113]. The real part of the complex geometrical nonadiabatic phase is given by

$$
\operatorname{Re}[\beta]= \begin{cases}\pi, & \text { Region (I) } \\ \pi\left[1+\frac{\Gamma_{-}}{\left.\sqrt{\Gamma_{-}^{2}-\gamma^{2}\left[1-(\omega-2 h)^{2} /\left(4 J^{2}\right)\right.}\right]}\right], & \text { Region (II) } \\ \pi[1-f(k)], & \text { Region (III) }\end{cases}
$$

where

$$
f(k)=\frac{2 h+2 J \cos (k)-\omega}{2 \operatorname{Re}[\Delta]}-\frac{\Gamma_{-}}{\operatorname{Im}[\Delta]} .
$$

As seen the real part of the complex geometrical nonadiabatic phase shows singularity at phase boundaries. In addition, the real part of the complex geometrical nonadiabatic phase in region (I), which is confined to exceptional points, is independent of the Hamiltonian parameters. In the next section we will study the topological properties of the effective Hamiltonian in Eq. (7) using the winding numbers of the non-Hermitian Hamiltonians [65].

\section{Topological invariant}

Examining the non-Hermitian Hamiltonians' winding numbers expresses that both inside and outside the exceptional ring is distinguished by its winding number, $\quad N_{w}=\frac{1}{2 \pi} \int_{-\pi}^{\pi} \partial_{k} \phi(k) d k$. Here $\phi(k)=\arctan [(\omega-$ $\left.\left.2 h_{z}(k)\right) /\left(2 h_{y}(k)\right)\right]$ is the winding angle [65]. The winding numbers of the non-Hermitian topological and trivial phases are found to be $N_{w}=1$ for $\left(\Gamma_{-}^{2} / \gamma^{2}\right)+\left[(\omega-2 h)^{2} /\left(4 J^{2}\right)\right]<1$ (inside the exceptional ring) and $N_{w}=0$ for outside the exceptional ring, respectively. As can be seen, the topological phase spreads by dissipation which is unique to non-Hermitian systems.

\section{Pure state dynamical topological quantum phase transition}

As obtained in Eq. (10), if at $t=0$ the system prepared at $\left|\psi_{-}(0)\right\rangle=|\downarrow\rangle$, the unnormalized time-evolved initial state of the dissipative Floquet $X Y$ Hamiltonian is expressed as

$$
\begin{aligned}
&\left|\psi_{-}(k, t)\right\rangle=\left[e^{-\Gamma_{+} t}\left(\frac{h_{x y}(k)}{\Lambda} \sin (\Lambda t)\right)|\uparrow\rangle+e^{-\Gamma_{+} t} e^{i \omega t}\right. \\
&\left.\quad \times\left(\cos (\Lambda t)+i \frac{2 h_{z}(k)-\omega}{2 \Lambda} \sin (\Lambda t)\right)|\downarrow\rangle\right]
\end{aligned}
$$

It is straightforward to see how the return probability (LA) is determined:

$$
\mathcal{L}(k, t)=e^{-\Gamma_{+} t} e^{i \omega t}\left[\frac{\cos (\Lambda t)+i \frac{2 h_{z}(k)-\omega}{2 \Lambda} \sin (\Lambda t)}{\sqrt{\left\langle\psi_{-}(k, t) \mid \psi_{-}(k, t)\right\rangle}}\right] .
$$

The FDQPT occurs at the time instances at which at least one factor in LA becomes zero, i.e., $\mathcal{L}_{k^{*}}\left(t^{*}\right)=0$ which yields

$$
t^{*}=\frac{-i}{2 \Lambda} \ln \left[\frac{2 h_{z}-\omega-2 \Lambda}{2 h_{z}-\omega+2 \Lambda}\right] .
$$


(a) $\Gamma_{u}=0 ; \Gamma_{d}=0$

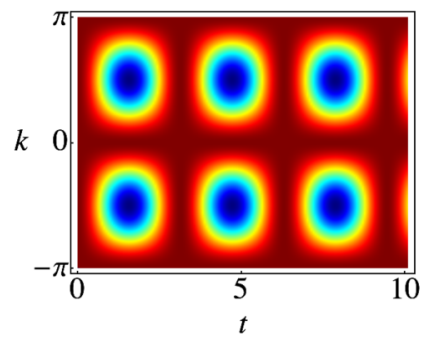

(e) $\Gamma_{u}=0 ; \Gamma_{d}=0$

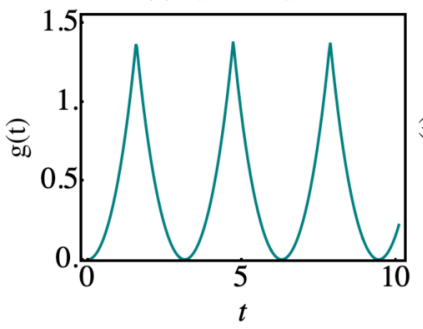

(i) $\Gamma_{u}=0 ; \Gamma_{d}=0$

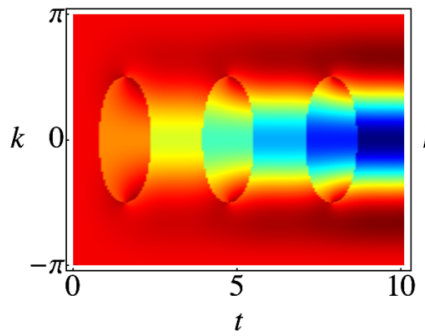

(b) $\Gamma_{u}=0.5 ; \Gamma_{d}=0.2$

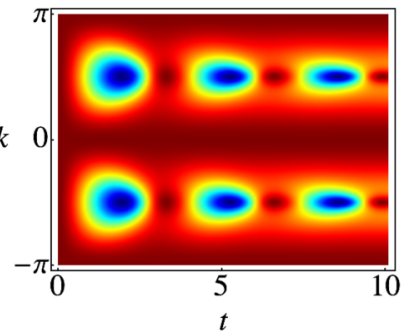

(f) $\Gamma_{u}=0.5 ; \Gamma_{d}=0.2$

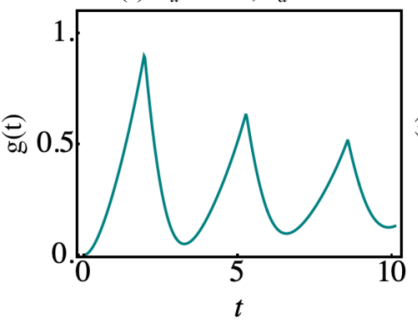

(j) $\Gamma_{u}=0.5 ; \Gamma_{d}=0.2$

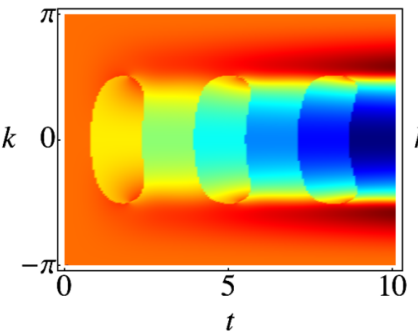

(c) $\Gamma_{u}=0.8 ; \Gamma_{d}=0.1$

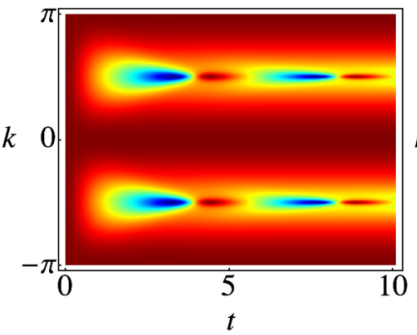

(g) $\Gamma_{u}=0.8 ; \Gamma_{d}=0.1$

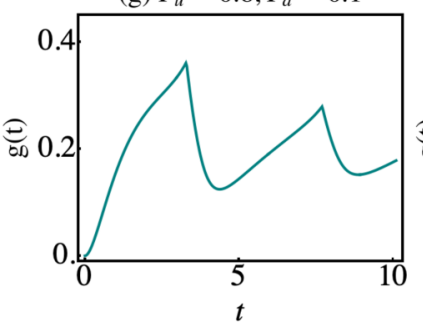

(k) $\Gamma_{u}=0.8 ; \Gamma_{d}=0.1$

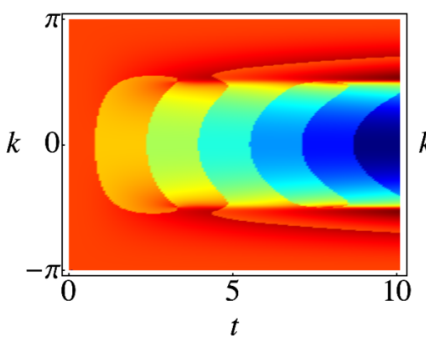

(d) $\Gamma_{u}=1.2 ; \Gamma_{d}=0.1$

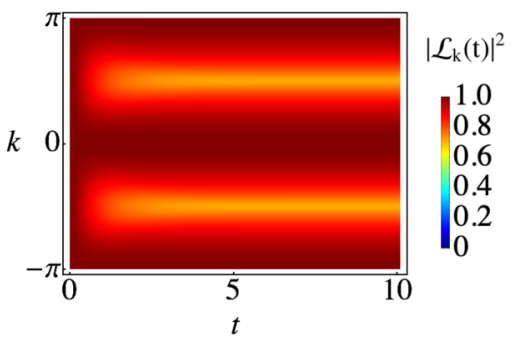

(h) $\Gamma_{u}=1.2 ; \Gamma_{d}=0.1$

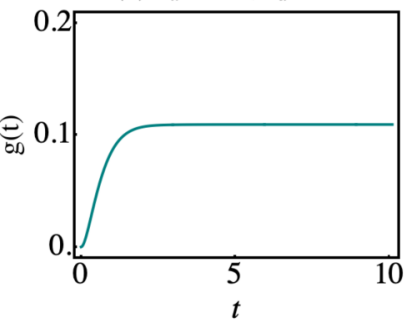

(1) $\Gamma_{u}=1.2 ; \Gamma_{d}=0.1$

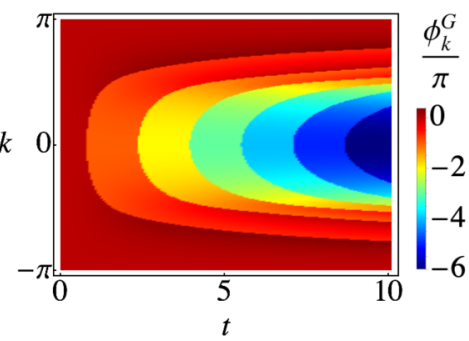

FIG. 2. The density plot of Loschmidt echo $\left|\mathcal{L}_{k}(t)\right|^{2}$ of the periodically time-driven $X Y$ model as a function of time $t$ and $k$, for (a) $\Gamma_{-}=0$, (b) $\Gamma_{-}=0.3$, (c) $\Gamma_{-}=0.7$, (d) $\Gamma_{-}=1.1$. The dynamical free energy of the model versus time $t$ for (e) $\Gamma_{-}=0$, (f) $\Gamma_{-}=0.3$, (g) $\Gamma_{-}=0.7$, (h) $\Gamma_{-}=1.1$. The density plot of geometric phase as a function of time and $k$ for (i) $\Gamma_{-}=0$, (j) $\Gamma_{-}=0.3$, (k) $\Gamma_{-}=0.7$, (l) $\Gamma_{-}=1.1$. In all plots we set $J=h=\gamma=1$ and $\omega=2$.

By a rather lengthy calculation, one can obtain that there are real solutions of $t^{*}$ only whenever

$$
2\left(h-J \sqrt{1-\frac{\Gamma_{-}^{2}}{\gamma^{2}}}\right)<\omega<2\left(h+J \sqrt{1-\frac{\Gamma_{-}^{2}}{\gamma^{2}}}\right),
$$

at quasimomentum $k^{*}=\arccos [(\omega-2 h) /(2 J)]$ results

$$
t^{*}=\frac{1}{2 \Lambda}(2 n+1) \pi+\frac{1}{\Lambda} \arctan \left(\frac{\Gamma_{-}}{\Lambda}\right) .
$$

DFDPTs arise in the range of driving frequency over which the eigenvalues of the time-independent effective Floquet nonHermitian $X Y$ Hamiltonian are purely real and the system is also topological, since Eq. (22) is nothing but Eq. (13). On the other hand, when $\Gamma_{-}^{2} / \gamma^{2}+(\omega-2 h)^{2} /\left(4 J^{2}\right)>1$, there is no critical momentum and $t^{*}$ is always complex resulting in no DFDPTs at any given real time $t$. We should note that the term $(2 n+1) \pi /(2 \Lambda)$ in Eq. (23) is the FDPT time scale in the absence of dissipation and the term $\left[\arctan \left(\Gamma_{-} / \Lambda\right)\right] / \Lambda$ originates from the dissipation. As is clear, both the lower and upper bounds of the range of driven frequency over which DFDPTs occur are functions of the dissipation. Thus, the DFDPT driven frequency range shrinks to a single point $\omega=2 h$ at $\Gamma_{-}= \pm \gamma$. When the gain or loss of the spin-up and spin-down states are equal, $\Gamma_{u}=\Gamma_{d} \neq 0$, the DFDPT times drop to nondissipative FDPT times even in the presence of dissipation, as shown by Eq. (23). In such a case, the system is in the resonance regime where the population completely cycles the population between the two spin-down and spinup states. It is worthwhile to mention that, the anisotropy $\gamma$ does not affect the nondissipative FDPTs driven frequency range $\left(\Gamma_{u}=\Gamma_{d}=0\right)$ [103-105], while the DFDPTs driven frequency range controls by $\gamma$.

The numerical simulation of the density plot of the Loschmidt echo $|\mathcal{L}(k, t)|^{2}$, the dynamical free energy $g(t)$, and density plot of the geometric phase have been depicted in Fig. 2 for the Hamiltonian parameters inside and outside the exceptional ring. When the time-independent effective non-Hermitian $X Y$ Hamiltonian $H_{F}(k)$ is in the non-Hermitian topological phase, it is apparent that there exist critical points $k^{*}$ and $t^{*}$, where $\mathcal{L}_{k^{*}}\left(t^{*}\right)$ becomes zero [Figs. 2(a)-2(c)]. Outside of the exceptional ring, however, there is no such critical point [Fig. 2(d)]. Moreover, in Figs. 2(e)-2(g) the DFDPTs are observed as the cusps in $g(t)$ for the driving frequency at which the system enters into the non-Hermitian topological phase, while the dynamical free energy shows completely analytic, smooth behavior for the Hamiltonian parameters set outside the exceptional ring.

The density plots of the geometric phase are plotted in Figs. 2(i)-2(1) for different values of Hamiltonian's parameters inside and outside of the exceptional ring. As seen, the plots display singular changes at critical times $t^{*}$, and 
at critical momentum $k^{*}$ when the system is in region (I), while it shows smooth behavior for the case that the DPTs are absent. This behavior represents the topological aspects of DFDPTs, where the phase of the time-independent effective Floquet non-Hermitian $X Y$ Hamiltonian is topological.

\section{DISSIPATIVE PERIODICALLY TIME-DRIVEN EXTENDED $X Y$ MODEL}

In this section we study the phase diagram, topological properties, and FDPTs of the dissipative periodically timedriven extended $X Y$ model. We show that the region where DFDPTs occur is confined to exceptional points and the time-independent effective Floquet non-Hermitian extended $X Y$ Hamiltonian has real eigenvalues but the system is topologically trivial.

\section{A. Exact solution}

The Hamiltonian of the one-dimensional harmonically driven extended $X Y$ spin chain in the staggered magnetic field is given by [103]

$$
\begin{aligned}
\mathcal{H}(t) & =\sum_{n=1}^{N}\left[J_{1} \cos (\omega t)\left(S_{n}^{x} S_{n+1}^{x}+S_{n}^{y} S_{n+1}^{y}\right)\right. \\
& -(-1)^{n} J_{1} \sin (\omega t)\left(S_{n}^{x} S_{n+1}^{y}-S_{n}^{y} S_{n+1}^{x}\right) \\
& -(-1)^{n} J_{2}\left(S_{n}^{x} S_{n+1}^{z} S_{n+2}^{x}+S_{n}^{y} S_{n+1}^{z} S_{n+2}^{y}\right) \\
& \left.-i\left(\Gamma_{u} S_{n}^{+} S_{n}^{-}+\Gamma_{d} S_{n}^{-} S_{n}^{+}\right)+(-1)^{n} h_{s} S_{n}^{z}\right] .
\end{aligned}
$$

The first and second terms in Eq. (24) describe the timedependent nearest neighbor $X Y$ and staggered DzyaloshinskiiMoriya interactions [114], and the third term is a staggered cluster (three-spin) interaction [115].

This Hamiltonian can be exactly diagonalized by JordanWigner transformation [107-110] which transforms spins into spinless fermions, where $c_{n}^{\dagger}\left(c_{n}\right)$ is the fermion creation (annihilation) operator [103]. The crucial step is to define two independent fermions at site $n, c_{n-1 / 2}^{A}=c_{2 n-1}$, and $c_{n}^{B}=c_{2 n}$, which can be regarded as splitting the chain having a diatomic unit cell. The Fourier transformed Hamiltonian can be expressed as the sum of independent terms $\mathcal{H}(t)=\sum_{k} \mathcal{H}_{k}(t)$ with $\mathcal{H}_{k}(t)=\Psi^{\dagger} H_{k}(t) \Psi-i \Gamma_{+} \mathbb{1}$ and $\Psi_{k}^{\dagger}=\left(c_{k}^{\dagger B}, c_{k}^{\dagger A}\right)$, where the Bloch single-particle Hamiltonian $\mathbb{H}_{k}(t)$ is given as $\mathrm{H}_{k}(t)=\left[h_{x y}\left(\cos (\omega t) \sigma^{x}+\sin (\omega t) \sigma^{y}\right)+h_{z} \sigma^{z}\right]$, with $h_{x y}(k)=$ $J_{1} \cos (k / 2)$ and $h_{z}(k)=J_{2} \cos (k) / 2+h_{s}-i \Gamma_{-}$.

Using the time-dependent Schrödinger equation $i \frac{d}{d t}\left|\psi_{k}^{ \pm}(t)\right\rangle=\uplus_{k}(t)\left|\psi_{k}^{ \pm}(t)\right\rangle$ in the rotating frame given by the periodic nonunitary transformation $U(t)=U_{R}(t) U_{D}(t)$, with $U_{R}(t)=\exp \left[i \omega\left(\mathbb{1}-\sigma^{z}\right) t / 2\right]$, and $U_{D}(t)=e^{-\Gamma_{+} t} \mathbb{1}$ the time-dependent Hamiltonian is transformed to the time-independent effective Floquet non-Hermitian form,

$$
H_{F}(k)=h_{x y}(k) \sigma^{x}+\left(h_{z}(k)-\frac{\omega}{2}\right) \sigma^{z}+\frac{\omega}{2} \mathbb{1} .
$$

Following the calculation in Sec. III A, if at $t=0$ the system prepared at $|\psi(0)\rangle=\left|\varphi_{k}\right\rangle=|\downarrow\rangle$, then according to Eqs. (8) and (9) the unnormalized time-evolving state $|\psi(k, t)\rangle$ of the
Hamiltonian $\mathcal{H}_{k}(t)$ is given by

$$
\begin{aligned}
|\psi(t)\rangle= & \prod_{k}|\psi(k, t)\rangle \\
|\psi(k, t)\rangle= & {\left[e^{-\Gamma_{+} t}\left(-i \frac{h_{x y}(k)}{\Lambda} \sin (\Lambda t)\right)|\uparrow\rangle+e^{-\Gamma_{+} t} e^{i \omega t}\right.} \\
& \left.\times\left(\cos (\Lambda t)+i \frac{2 h_{z}(k)-\omega}{2 \Lambda} \sin (\Lambda t)\right)|\downarrow\rangle\right]
\end{aligned}
$$

with $\Lambda=\sqrt{h_{x y}^{2}(k)+\left[h_{z}(k)-\frac{\omega}{2}\right]^{2}}$.

The complex energy spectrum of $H_{F}(k)$ is given as

$$
\epsilon_{k}^{ \pm}=\frac{\omega}{2} \pm \sqrt{h_{x y}^{2}(k)+\left[h_{z}(k)-\frac{\omega}{2}\right]^{2}}
$$

and become gapless if

$$
\begin{aligned}
\Gamma_{-}\left[J_{2} \cos (k)+2 h_{s}-\omega\right] & =0, \\
\frac{1}{4}\left[J_{2} \cos (k)+2 h_{s}-\omega\right]^{2}+\left[J_{1} \cos \left(\frac{k}{2}\right)\right]^{2}-\Gamma_{-} & =0 .
\end{aligned}
$$

By solving these equations, we can get

$$
\begin{aligned}
& k^{*}=\arccos \left(\frac{\omega-2 h_{s}}{J_{2}}\right), \\
& \frac{2 \Gamma_{-}^{2}}{J_{1}^{2}}-\frac{\omega-2 h_{s}}{J_{2}}=1 .
\end{aligned}
$$

The first term of Eq. (28) implies a limitation $\omega-2 h_{s}< \pm J_{2}$, and the second one defines exceptional points. Therefore, the system can be separated into three regions as shown in Fig. 3. In region (I), inside the exceptional closed curve, the energy gap $\Delta=\left|\epsilon_{K}^{+}-\epsilon_{k}^{-}\right|$is purely real, i.e., $\operatorname{Im}[\Delta]=$ 0 and $\operatorname{Re}[\Delta]>0$. In this region $k^{*}=\arccos \left[\left(\omega-2 h_{s}\right) / J_{2}\right]$ and $\Delta=\sqrt{J_{1}^{2}\left(J_{2}+\omega-2 h_{s}\right) /\left(2 J_{2}\right)-\Gamma_{-}^{2}}$. In region (II) the gap is pure imaginary, i.e., $\operatorname{Im}[\Delta] \neq 0$ and $\operatorname{Re}[\Delta]=0$. In this region we still have $k^{*}=\arccos \left[\left(\omega-2 h_{s}\right) / J_{2}\right]$ but the non-Hermitian strength $\Gamma_{-}$is large enough to be dominant, then $\Delta=i \sqrt{\Gamma_{-}^{2}-J_{1}^{2}\left(J_{2}+\omega-2 h_{s}\right) /\left(2 J_{2}\right)}$. Region (III) $\left(\left|\omega-2 h_{s}\right|>J_{2}\right)$ is characterized by the complex gap. In other words, in region (III) both real and imaginary parts of the gap are nonzero.

According to discussion in Sec. III B, the complex geometrical nonadiabatic phase for the periodically time-driven Floquet extended $X Y$ model is also given by Eq. (18), in which $h_{x y}(k)=J_{1} \cos (k / 2)$ and $h_{z}(k)=J_{2} \cos (k) / 2+h_{s}-$ $i \Gamma_{\text {- }}$. Then the real part of the complex geometrical nonadiabatic phase is given by

$$
\operatorname{Re}[\beta]= \begin{cases}\pi, & \text { Region (I) } \\ \pi\left[1+\frac{\Gamma_{-}}{\sqrt{\Gamma_{-}^{2}-J_{1}^{2}\left(J_{2}+\omega-2 h_{s}\right) /\left(2 J_{2}\right)}}\right], & \text { Region (II) } \\ \pi[1-f(k)], & \text { Region (III) }\end{cases}
$$

where

$$
f(k)=\left(\left[2 h_{s}+J_{2} \cos (k)-\omega\right] /(2 \operatorname{Re}[\Delta])\right)-\left(\Gamma_{-} / \operatorname{Im}[\Delta]\right) .
$$




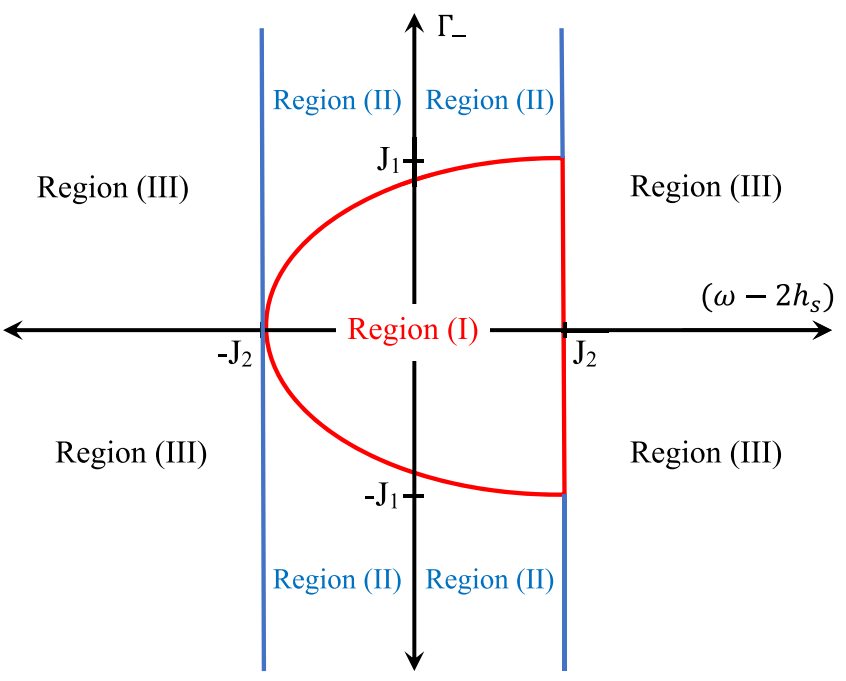

FIG. 3. The phase diagram of the time-independent effective Floquet non-Hermitian extended $X Y$ Hamiltonian. The red line denotes the exceptional ring, which corresponds to Eq. (28). In region (I), the eigenvalues (gap) of the time-independent effective Floquet nonHermitian extended $X Y$ Hamiltonian is purely real. In region (II) the energy gap of the effective non-Hermitian Hamiltonian is a pure imaginary. In region (III) the energy gap is complex.

As seen the real part of the complex geometrical nonadiabatic phase shows singularity at phase boundaries. It is necessary to mention that all regions in Fig. 4 are topologically trivial and the winding number is zero.

\section{B. Pure state dynamical topological quantum phase transition}

The Loschmidt amplitude for extended $X Y$ model is calculated as

$$
\mathcal{L}(k, t)=e^{-\Gamma_{+} t} e^{i \omega t}\left[\frac{\cos (\Lambda t)+i \frac{2 h_{z}(k)-\omega}{2 \Lambda} \sin (\Lambda t)}{\sqrt{\left\langle\psi_{-}(k, t) \mid \psi_{-}(k, t)\right\rangle}}\right] .
$$

The DQPT occurs at the time instances at which at least one factor in LA becomes zero, i.e., $\mathcal{L}_{k^{*}}\left(t^{*}\right)=0$ which yields

$$
t^{*}=\frac{-i}{2 \Lambda} \ln \left[\frac{2 h_{z}-\omega-2 \Lambda}{2 h_{z}-\omega+2 \Lambda}\right] .
$$

It is straightforward to show that there are real solutions of $t^{*}$ only whenever

$$
2 h_{s}+J_{2}\left(\frac{2 \Gamma_{-}^{2}}{J_{1}^{2}}-1\right)<\omega<2 h_{s}+J_{2}
$$

(a) $\Gamma_{u}=0 ; \Gamma_{d}=0$

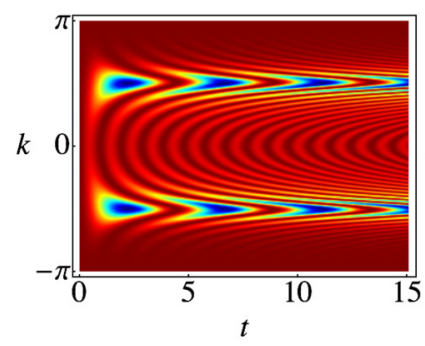

(e) $\Gamma_{u}=0 ; \Gamma_{d}=0$

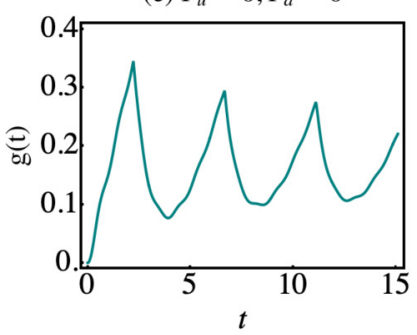

(i) $\Gamma_{u}=0 ; \Gamma_{d}=0$

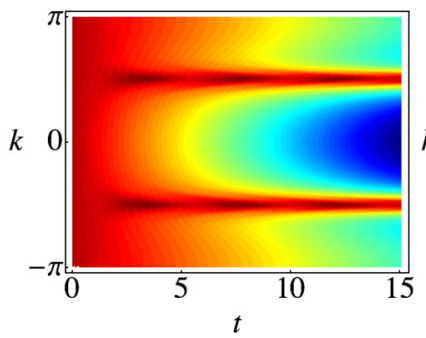

(b) $\Gamma_{u}=0.5 ; \Gamma_{d}=0.2$

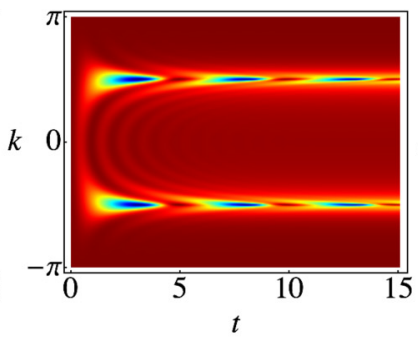

(f) $\Gamma_{u}=0.5 ; \Gamma_{d}=0.2$

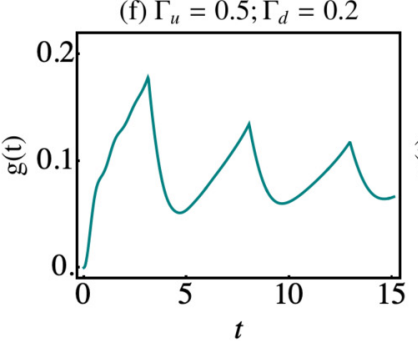

(j) $\Gamma_{u}=0.5 ; \Gamma_{d}=0.2$

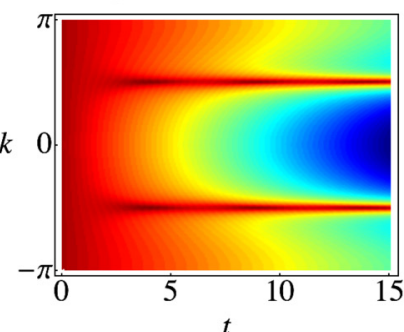

(c) $\Gamma_{u}=0.8 ; \Gamma_{d}=0.3$

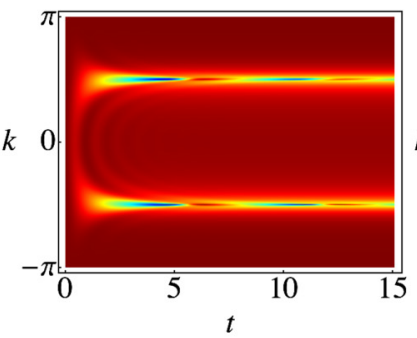

(g) $\Gamma_{u}=0.8 ; \Gamma_{d}=0.3$

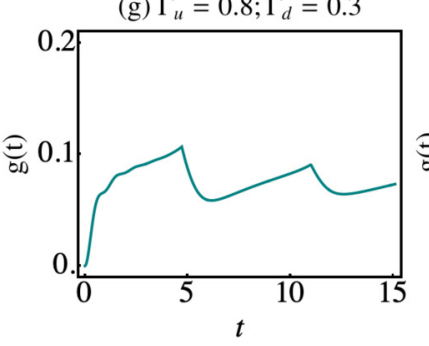

(k) $\Gamma_{u}=0.8 ; \Gamma_{d}=0.3$

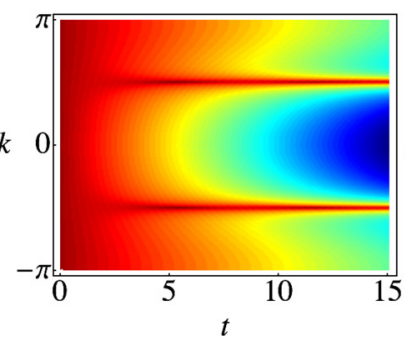

(d) $\Gamma_{u}=1.2 ; \Gamma_{d}=0.1$

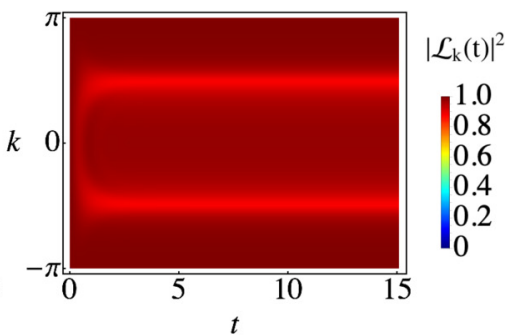

(h) $\Gamma_{u}=1.2 ; \Gamma_{d}=0.1$

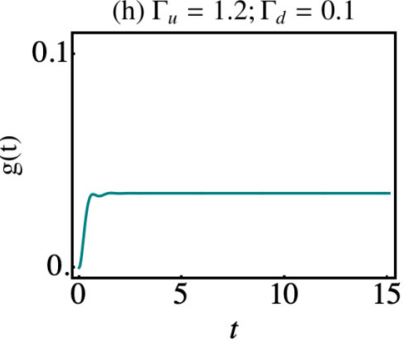

(1) $\Gamma_{u}=1.2 ; \Gamma_{d}=0.1$

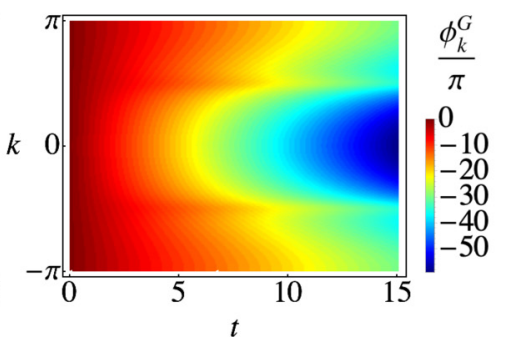

FIG. 4. The density plot of Loschmidt echo $\left|\mathcal{L}_{k}(t)\right|^{2}$ of the periodically time-driven extended $X Y$ model as a function of time $t$ and $k$, for (a) $\Gamma_{-}=0$, (b) $\Gamma_{-}=0.3$, (c) $\Gamma_{-}=0.5$, (d) $\Gamma_{-}=1.1$. The dynamical free energy of the model versus time $t$ for (e) $\Gamma_{-}=0$, (f) $\Gamma_{-}=0.3$, (g) $\Gamma_{-}=0.5$, (h) $\Gamma_{-}=1.1$. The density plot of the geometric phase as a function of time and $k$ for (i) $\Gamma_{-}=0,(\mathrm{j}) \Gamma_{-}=0.3$, (k) $\Gamma_{-}=0.5$, (l) $\Gamma_{-}=1.1$. In all plots we take $J_{1}=1, J_{2}=2 \pi, h_{s}=3 \pi, \omega=6 \pi$. 
at quasimomentum $k^{*}=\arccos \left[\left(\omega-2 h_{s}\right) / J_{2}\right]$ results in

$$
t^{*}=\frac{1}{2 \Lambda}(2 n+1) \pi+\frac{1}{\Lambda} \arctan \left(\frac{\Gamma_{-}}{\Lambda}\right) .
$$

According to Eq. (31) or Eq. (28), DFDPTs exist in the range of driving frequency over which the eigenvalues of the timeindependent effective Floquet non-Hermitian Hamiltonian are purely real but the system is not topological. There is no critical momentum when $\left(\Gamma_{-}^{2} / J_{1}^{2}\right)-\left[\left(\omega-2 h_{s}\right) / J_{2}\right]>1$ and $t^{*}$ is always imaginary, resulting in no DFDPTs at any real time $t$. The lower bound of the driven frequency range across which DFDPTs occur is clearly reliant on dissipation, but the upper bound is independent of dissipation coupling. Therefore, the range of driven frequency over which DFDPTs occur shrinks to a single point $\omega=J_{2}+2 h_{s}$ at $\Gamma_{-}= \pm J_{1}$. It is worth noting that, in the absence of dissipation, FDPTs do not rely on the exchange coupling $J_{1}$, however, in the presence of dissipation, the DFDPT driven frequency range depends on $J_{1}$.

We present the density plot of the Loschmidt echo $|\mathcal{L}(k, t)|^{2}$, the dynamical free energy $g(t)$, and the density plot of geometric phase in Fig. (4) for different values of dissipation. Figures 4(a)-4(c) show that when the eigenvalues of the time-independent effective Floquet non-Hermitian extended $X Y$ Hamiltonian $H_{F}(k)$ are pure real, region (I), there exist critical points $k^{*}$ and $t^{*}$, where $\mathcal{L}_{k^{*}}\left(t^{*}\right)$ becomes zero. However, there is no such critical point outside of region (I) [Fig. 4(d)]. Moreover, Figs. 4(e)-4(h) observe DFDPTs as cusps in $g(t)$ for the driving frequency at which the system enters into region (I), while $g(t)$ shows completely analytic, smooth behavior when the Hamiltonian parameters set outside region (I).

The density plots of $\Phi_{k}^{G}$ are also plotted in Figs. 4(i)-4(1) for different values of the Hamiltonian's parameters inside and outside of region (I). As seen, the plots display singular changes at critical times $t^{*}$, and at critical momentum $k^{*}$ when the system is in region (I), while it shows smooth behavior for the case that the DFDPTs are absent. This behavior represents the topological aspects of DFDPTs, where the phase of the time-independent effective Floquet non-Hermitian extended $X Y$ Hamiltonian is not topological.

In is remarkable to mention that, in the absence of the dissipation, the lower bound of the driven frequency range in Eq. (31) and both lower and upper bounds of the driven frequency range in Eq. (22) are the critical points (gap closing) of the time-independent effective Floquet Hermitian Hamiltonians in Eqs. (25) and (7). However, the upper bound of the driven frequency range in Eq. (22) is not the critical point of the time-independent effective Floquet Hermitian Hamiltonian in Eq. (25). As a result, we may conclude that, in the absence of dissipation, only the gap closing (critical) points of the time-independent effective Floquet Hermitian Hamiltonian are affected by dissipation.

\section{CONCLUSION}

We have investigated the dissipative Floquet dynamical phase transition in the periodically time-driven $X Y$ and extended $X Y$ models in the presence of the imaginary terms, which represent the physical gain and loss during the interacting processes with the environment. We have shown that the time-independent effective Floquet non-Hermitian Hamiltonians reveal three regions with pure real eigenvalues (gap) where confined to exceptional points, pure imaginary gap, and complex gap. We have found that the complex geometrical nonadiabatic phase can distinguish each region of the system. We have shown that the Floquet dynamical phase transitions still appear in the presence of the dissipation in the region where the time-independent effective Floquet non-Hermitian Hamiltonians exhibit real eigenvalues. While the real gap region in the time-independent effective Floquet non-Hermitian $X Y$ Hamiltonian is topologically nontrivial, its counterpart in the time-independent effective Floquet non-Hermitian extended $X Y$ Hamiltonian is topologically trivial. In other words, different from results obtained for the quenched case, existence of the non-Hermitian topologically nontrivial phase is not a necessary condition for appearance of the dissipative Floquet dynamical phase transitions. We have also shown that the range of driven frequency, over which the dissipative Floquet dynamical phase transitions occur, narrows down by increasing the dissipation coupling and shrinks to a single point at the critical value of dissipation. Furthermore, the topological characteristic aspect of the dissipative Floquet dynamical phase transitions in the real gap region is revealed by quantization and jumps of the dynamical geometric phase.

\section{ACKNOWLEDGMENTS}

A.A. acknowledges the support of the Max PlanckPOSTECH-Hsinchu Center for Complex Phase Materials, and financial support from the National Research Foundation (NRF) funded by the Ministry of Science of Korea (Grant No. 2016K1A4A01922028).

\section{APPENDIX A: SPINLESS FERMION TRANSFORMATION OF THE FLOQUET $X Y$ MODEL}

The Hamiltonian, Eq. (1), can be diagonalized using the Jordan-Wigner transformation [107-110],

$$
\begin{aligned}
& S_{n}^{+}=S_{n}^{x}+i S_{n}^{y}=\prod_{m=1}^{n-1}\left(1-2 c_{m}^{\dagger} c_{m}\right) c_{n}^{\dagger}, \\
& S_{n}^{-}=S_{n}^{x}-i S_{n}^{y}=\prod_{m=1}^{n-1} c_{n}\left(1-2 c_{m}^{\dagger} c_{m}\right), \\
& S_{n}^{z}=c_{n}^{\dagger} c_{n}-\frac{1}{2},
\end{aligned}
$$

which transforms spins into fermion operators $c_{n}$ and $c_{n}^{\dagger}$. Using the Fourier transform, the Hamiltonian of Eq. (2) can be written as the sum of $N / 2$ noninteracting terms,

$$
\mathcal{H}(t)=\sum_{k>0} \mathcal{H}_{k}(t),
$$

where this local Hamiltonian reads

$$
\begin{aligned}
\mathcal{H}_{k}(t) & =\left[J \cos (k)+h-i \Gamma_{-}\right]\left(c_{k}^{\dagger} c_{k}+c_{-k}^{\dagger} c_{-k}\right) \\
& -i \gamma \sin (k)\left(e^{i \omega t} c_{k} c_{-k}-e^{-i \omega t} c_{k}^{\dagger} c_{-k}^{\dagger}\right)-i \Gamma_{+},
\end{aligned}
$$

where the wave number $k$ is equal to $k=(2 p-1) \pi / N$ and $p$ runs from 1 to $N / 2$. By defining the fermionic two-component 
spinor $C^{\dagger}=\left(c_{k}^{\dagger}, c_{-k}\right)$ the Hamiltonian $\mathcal{H}(t)$ can be written as $\mathcal{H}_{k}(t)=C^{\dagger} \uplus_{k}(t) C-i \Gamma_{+} \mathbb{1}$, where $\uplus_{k}(t)$ is given by Eq. (6). We can get the eigenvalues and eigenvectors of Hamiltonian $\mathcal{H}_{k}(t)$ by solving the time-dependent Schrödinger equation:

$$
i \frac{d}{d t}|\psi(k, t)\rangle=\mathcal{H}_{k}(t)|\psi(k, t)\rangle
$$

The exact solution to the Schrödinger equation is found by going to the rotating frame given by the nonunitary transformation $U(t)=U_{R}(t) U_{D}(t)$, with

$$
U_{R}(t)=\left(\begin{array}{cc}
1 & 0 \\
0 & e^{i \omega t}
\end{array}\right), \quad U_{D}(t)=\left(\begin{array}{cc}
e^{-\Gamma_{+} t} & 0 \\
0 & e^{-\Gamma_{+} t}
\end{array}\right) .
$$

In the rotated frame the eigenstate is given by $|\varphi(k)\rangle=$ $U^{-1}(t)|\psi(k, t)\rangle$. Substituting the transformed eigenstate into the Schrödinger equation, we can obtain the time-independent effective Flouquet non-Hermitian Hamiltonian:

$$
i \frac{d}{d t}|\varphi(k)\rangle=\left[U^{-1}(t) \mathcal{H}_{k}(t) U(t)-i U^{-1}(t) \frac{d U(t)}{d t}\right]|\varphi(k)\rangle .
$$

Under this unitary transformation the time-independent effective Flouquet non-Hermitian Hamiltonian $H_{F}(k)$ is given by Eq. (7).

\section{APPENDIX B: COMPLEX GEOMETRICAL NONADIABATIC PHASE}

The instantaneous eigenstates of $I(t)$ and $I(t)^{\dagger}$ are given as

$$
\left|\Psi_{+}\right\rangle=\left(\begin{array}{c}
\cos \left(\frac{\alpha}{2}\right) \\
\sin \left(\frac{\alpha}{2}\right) e^{i \omega t}
\end{array}\right), \quad\left|\Psi_{-}\right\rangle=\left(\begin{array}{c}
-\sin \left(\frac{\alpha}{2}\right) e^{-i \omega t} \\
\cos \left(\frac{\alpha}{2}\right)
\end{array}\right)
$$

and

$$
\begin{aligned}
& \left\langle\Phi_{+}\right|=\left(\cos \left(\frac{\alpha}{2}\right) \quad \sin \left(\frac{\alpha}{2}\right) e^{-i \omega t}\right), \\
& \left\langle\Phi_{-}\right|=\left(-\sin \left(\frac{\alpha}{2}\right) e^{i \omega t} \quad \cos \left(\frac{\alpha}{2}\right)\right),
\end{aligned}
$$

respectively.
[1] R. Hamazaki, Nat. Commun. 12, 5108 (2021).

[2] J. Li, A. K. Harter, J. Liu, L. de Melo, Y. N. Joglekar, and L. Luo, Nat. Commun. 10, 855 (2019).

[3] J. Doppler, A. A. Mailybaev, J. Böhm, U. Kuhl, A. Girschik, F. Libisch, T. J. Milburn, P. Rabl, N. Moiseyev, and S. Rotter, Nature (London) 537, 76 (2016).

[4] R. El-Ganainy, K. G. Makris, M. Khajavikhan, Z. H. Musslimani, S. Rotter, and D. N. Christodoulides, Nat. Phys. 14, 11 (2018).

[5] L. Lu, J. D. Joannopoulos, and M. Soljačić, Nat. Photonics 8 , 821 (2014).

[6] W. Chen, Ş. K. Özdemir, G. Zhao, J. Wiersig, and L. Yang, Nature (London) 548, 192 (2017).

[7] H. Xu, D. Mason, L. Jiang, and J. Harris, Nature (London) 537, 80 (2016).

[8] L. Feng, Z. J. Wong, R.-M. Ma, Y. Wang, and X. Zhang, Science 346, 972 (2014).

[9] H. Hodaei, M.-A. Miri, M. Heinrich, D. N. Christodoulides, and M. Khajavikhan, Science 346, 975 (2014).

[10] H. Hodaei, A. U. Hassan, S. Wittek, H. Garcia-Gracia, R. ElGanainy, D. N. Christodoulides, and M. Khajavikhan, Nature (London) 548, 187 (2017).

[11] B. Peng, Ş. Özdemir, S. Rotter, H. Yilmaz, M. Liertzer, F. Monifi, C. Bender, F. Nori, and L. Yang, Science 346, 328 (2014).

[12] M. Liertzer, L. Ge, A. Cerjan, A. D. Stone, H. E. Türeci, and S. Rotter, Phys. Rev. Lett. 108, 173901 (2012).

[13] L. Feng, Y.-L. Xu, W. S. Fegadolli, M.-H. Lu, J. E. Oliveira, V. R. Almeida, Y.-F. Chen, and A. Scherer, Nat. Mater. 12, 108 (2013).

[14] T. Ozawa, H. M. Price, A. Amo, N. Goldman, M. Hafezi, L. Lu, M. C. Rechtsman, D. Schuster, J. Simon, O. Zilberberg, and I. Carusotto, Rev. Mod. Phys. 91, 015006 (2019).
[15] V. V. Konotop, J. Yang, and D. A. Zezyulin, Rev. Mod. Phys. 88, 035002 (2016).

[16] C. E. Rüter, K. G. Makris, R. El-Ganainy, D. N. Christodoulides, M. Segev, and D. Kip, Nat. Phys. 6, 192 (2010).

[17] A. Regensburger, C. Bersch, M.-A. Miri, G. Onishchukov, D. N. Christodoulides, and U. Peschel, Nature (London) 488, 167 (2012).

[18] S. Diehl, E. Rico, M. A. Baranov, and P. Zoller, Nat. Phys. 7, 971 (2011).

[19] S. Longhi, Phys. Rev. Lett. 105, 013903 (2010).

[20] H. J. Carmichael, Phys. Rev. Lett. 70, 2273 (1993).

[21] T. E. Lee, F. Reiter, and N. Moiseyev, Phys. Rev. Lett. 113, 250401 (2014).

[22] S. Malzard, C. Poli, and H. Schomerus, Phys. Rev. Lett. 115, 200402 (2015).

[23] K. G. Makris, R. El-Ganainy, D. N. Christodoulides, and Z. H. Musslimani, Phys. Rev. Lett. 100, 103904 (2008).

[24] S. Klaiman, U. Günther, and N. Moiseyev, Phys. Rev. Lett. 101, 080402 (2008).

[25] J. Y. Lee, J. Ahn, H. Zhou, and A. Vishwanath, Phys. Rev. Lett. 123, 206404 (2019).

[26] Y. Ashida and M. Ueda, Phys. Rev. Lett. 120, 185301 (2018).

[27] C. M. Bender, Rep. Prog. Phys. 70, 947 (2007).

[28] C. M. Bender, Contemp. Phys. 46, 277 (2005).

[29] J. Dalibard, Y. Castin, and K. Mølmer, Phys. Rev. Lett. 68, 580 (1992).

[30] M. B. Plenio and P. L. Knight, Rev. Mod. Phys. 70, 101 (1998).

[31] R. Dum, P. Zoller, and H. Ritsch, Phys. Rev. A 45, 4879 (1992).

[32] K. Mølmer, Y. Castin, and Jean Dalibard, J. Opt. Soc. Am. B 10, 524 (1993).

[33] G. Dattoli, T. Hermsen, L. Mezi, A. Renieri, and A. Torre, Phys. Rev. A 37, 4334 (1988). 
[34] Y. V. Kartashov and D. V. Skryabin, Phys. Rev. Lett. 122, 083902 (2019).

[35] M. A. Bandres, S. Wittek, G. Harari, M. Parto, J. Ren, M. Segev, D. N. Christodoulides, and M. Khajavikhan, Science 359 (2018).

[36] G. Harari, M. A. Bandres, Y. Lumer, M. C. Rechtsman, Y. D. Chong, M. Khajavikhan, D. N. Christodoulides, and M. Segev, Science 359 (2018).

[37] S. Liu, S. Ma, C. Yang, L. Zhang, W. Gao, Y. J. Xiang, T. J. Cui, and S. Zhang, Phys. Rev. Applied 13, 014047 (2020).

[38] T. Helbig, T. Hofmann, S. Imhof, M. Abdelghany, T. Kiessling, L. Molenkamp, C. Lee, A. Szameit, M. Greiter, and R. Thomale, Nat. Phys. 16, 747 (2020).

[39] T. Hofmann, T. Helbig, F. Schindler, N. Salgo, M. Brzezińska, M. Greiter, T. Kiessling, D. Wolf, A. Vollhardt, A. Kabaši, C. H. Lee, A. Bilušić, R. Thomale, and T. Neupert, Phys. Rev. Research 2, 023265 (2020).

[40] A. Siegman, Opt. Commun. 31, 369 (1979).

[41] H. C. Baker, Phys. Rev. Lett. 50, 1579 (1983).

[42] N. Moiseyev, Non-Hermitian Quantum Mechanics (Cambridge University Press, Cambridge, 2011).

[43] Y. Wu, W. Liu, J. Geng, X. Song, X. Ye, C.-K. Duan, X. Rong, and J. Du, Science 364, 878 (2019).

[44] W. Zhang, X. Ouyang, X. Huang, X. Wang, H. Zhang, Y. Yu, X. Chang, Y. Liu, D.-L. Deng, and L.-M. Duan, Phys. Rev. Lett. 127, 090501 (2021).

[45] J. Wiersig, Phys. Rev. Lett. 112, 203901 (2014).

[46] H.-K. Lau and A. A. Clerk, Nat. Commun. 9, 4320 (2018).

[47] Z. Lin, H. Ramezani, T. Eichelkraut, T. Kottos, H. Cao, and D. N. Christodoulides, Phys. Rev. Lett. 106, 213901 (2011).

[48] S. Yao and Z. Wang, Phys. Rev. Lett. 121, 086803 (2018).

[49] S. Yao, F. Song, and Z. Wang, Phys. Rev. Lett. 121, 136802 (2018).

[50] C. M. Bender and S. Boettcher, Phys. Rev. Lett. 80, 5243 (1998).

[51] D.-W. Zhang, Y.-L. Chen, G.-Q. Zhang, L.-J. Lang, Z. Li, and S.-L. Zhu, Phys. Rev. B 101, 235150 (2020).

[52] P. He, J.-H. Fu, D.-W. Zhang, and S.-L. Zhu, Phys. Rev. A 102, 023308 (2020).

[53] H. Shen, B. Zhen, and L. Fu, Phys. Rev. Lett. 120, 146402 (2018).

[54] E. J. Bergholtz, J. C. Budich, and F. K. Kunst, Rev. Mod. Phys. 93, 015005 (2021).

[55] K. Kawabata, K. Shiozaki, M. Ueda, and M. Sato, Phys. Rev. X 9, 041015 (2019).

[56] H. Shen and L. Fu, Phys. Rev. Lett. 121, 026403 (2018).

[57] M. Papaj, H. Isobe, and L. Fu, Phys. Rev. B 99, 201107(R) (2019).

[58] D.-W. Zhang, L.-Z. Tang, L.-J. Lang, H. Yan, and S.-L. Zhu, Sci. China-Phys. Mech. Astron. 63, 267062 (2020).

[59] L.-Z. Tang, L.-F. Zhang, G.-Q. Zhang, and D.-W. Zhang, Phys. Rev. A 101, 063612 (2020).

[60] H. Jiang, L.-J. Lang, C. Yang, S.-L. Zhu, and S. Chen, Phys. Rev. B 100, 054301 (2019).

[61] Y. Ashida, Z. Gong, and M. Ueda, Adv. Phys. 69, 249 (2020).

[62] Y. Pará, G. Palumbo, and T. Macrì, Phys. Rev. B 103, 155417 (2021).

[63] L. Zhou, Q.-h. Wang, H. Wang, and J. Gong, Phys. Rev. A 98, 022129 (2018).
[64] L. Zhou and Q. Du, New J. Phys. 23, 063041 (2021).

[65] B. Zhu, Y. Ke, H. Zhong, and C. Lee, Phys. Rev. Research 2, 023043 (2020).

[66] L. Zhou and J. Gong, Phys. Rev. B 98, 205417 (2018).

[67] L. Zhou, Phys. Rev. B 100, 184314 (2019).

[68] L. Zhou, Y. Gu, and J. Gong, Phys. Rev. B 103, L041404 (2021).

[69] L. Zhou and J. Pan, Phys. Rev. A 100, 053608 (2019).

[70] J. Pan and L. Zhou, Phys. Rev. B 102, 094305 (2020).

[71] M. Heyl, A. Polkovnikov, and S. Kehrein, Phys. Rev. Lett. 110, 135704 (2013).

[72] P. Jurcevic, H. Shen, P. Hauke, C. Maier, T. Brydges, C. Hempel, B. P. Lanyon, M. Heyl, R. Blatt, and C. F. Roos, Phys. Rev. Lett. 119, 080501 (2017).

[73] F. Andraschko and J. Sirker, Phys. Rev. B 89, 125120 (2014).

[74] J. C. Budich and M. Heyl, Phys. Rev. B 93, 085416 (2016).

[75] M. Heyl, Rep. Prog. Phys. 81, 054001 (2018).

[76] M. Abdi, Phys. Rev. B 100, 184310 (2019).

[77] N. Sedlmayr, P. Jaeger, M. Maiti, and J. Sirker, Phys. Rev. B 97, 064304 (2018).

[78] N. Sedlmayr, M. Fleischhauer, and J. Sirker, Phys. Rev. B 97, 045147 (2018).

[79] T. Masłowski and N. Sedlmayr, Phys. Rev. B 101, 014301 (2020).

[80] R. Jafari, H. Johannesson, A. Langari, and M. A. MartinDelgado, Phys. Rev. B 99, 054302 (2019).

[81] S. Bhattacharjee and A. Dutta, Phys. Rev. B 97, 134306 (2018).

[82] U. Bhattacharya, S. Bandyopadhyay, and A. Dutta, Phys. Rev. B 96, 180303(R) (2017).

[83] M. Heyl and J. C. Budich, Phys. Rev. B 96, 180304(R) (2017).

[84] X.-Y. Guo, C. Yang, Y. Zeng, Y. Peng, H.-K. Li, H. Deng, Y.-R. Jin, S. Chen, D. Zheng, and H. Fan, Phys. Rev. Applied 11, 044080 (2019).

[85] T. Tian, Y. Ke, L. Zhang, S. Lin, Z. Shi, P. Huang, C. Lee, and J. Du, Phys. Rev. B 100, 024310 (2019).

[86] K. Wang, X. Qiu, L. Xiao, X. Zhan, Z. Bian, W. Yi, and P. Xue, Phys. Rev. Lett. 122, 020501 (2019).

[87] P. Uhrich, N. Defenu, R. Jafari, and J. C. Halimeh, Phys. Rev. B 101, 245148 (2020).

[88] U. Mishra, R. Jafari, and A. Akbari, J. Phys. A: Math. Theor. 53, 375301 (2020).

[89] R. Jafari, Sci. Rep. 9, 2871 (2019).

[90] M. Sadrzadeh, R. Jafari, and A. Langari, Phys. Rev. B 103, 144305 (2021).

[91] W. C. Yu, P. D. Sacramento, Y. C. Li, and H.-Q. Lin, Phys. Rev. B 104, 085104 (2021).

[92] B. Zhou, Y. Zeng, and S. Chen, Phys. Rev. B 104, 094311 (2021).

[93] S. Porta, F. Cavaliere, M. Sassetti, and N. T. Ziani, Sci. Rep. 10, 12766 (2020).

[94] A. Sehrawat, C. Srivastava, and U. Sen, Phys. Rev. B 104, 085105 (2021).

[95] R. Modak and D. Rakshit, Phys. Rev. B 103, 224310 (2021).

[96] S. Peotta, F. Brange, A. Deger, T. Ojanen, and C. Flindt, Phys. Rev. X 11, 041018 (2021).

[97] J. J. Mendoza-Arenas, arXiv:2109.7709.

[98] T. H. Kyaw, V. M. Bastidas, J. Tangpanitanon, G. Romero, and L.-C. Kwek, Phys. Rev. A 101, 012111 (2020). 
[99] H. Lang, Y. Chen, Q. Hong, and H. Fan, Phys. Rev. B 98, 134310 (2018).

[100] A. Kosior, A. Syrwid, and K. Sacha, Phys. Rev. A 98, 023612 (2018).

[101] A. Kosior and K. Sacha, Phys. Rev. A 97, 053621 (2018).

[102] L. Zhou and Q. Du, J. Phys.: Condens. Matter 33, 345403 (2021).

[103] S. Zamani, R. Jafari, and A. Langari, Phys. Rev. B 102, 144306 (2020).

[104] R. Jafari and A. Akbari, Phys. Rev. A 103, 012204 (2021).

[105] K. Yang, L. Zhou, W. Ma, X. Kong, P. Wang, X. Qin, X. Rong, Y. Wang, F. Shi, J. Gong, and J. Du, Phys. Rev. B 100, 085308 (2019).
[106] Q.-B. Zeng, B. Zhu, S. Chen, L. You, and R. Lü, Phys. Rev. A 94, 022119 (2016).

[107] E. Lieb, T. Schultz, and D. Mattis, Ann. Phys. 16, 407 (1961). [108] E. Barouch and B. M. McCoy, Phys. Rev. A 3, 786 (1971).

[109] R. Jafari, Phys. Rev. B 84, 035112 (2011).

[110] R. Jafari, Eur. Phys. J. B 85, 167 (2012).

[111] H. R. Lewis and W. B. Riesenfeld, J. Math. Phys. 10, 1458 (1969).

[112] X.-C. Gao, J.-B. Xu, and T.-Z. Qian, Phys. Rev. A 46, 3626 (1992).

[113] J. Garrison and E. Wright, Phys. Lett. A 128, 177 (1988).

[114] R. Jafari and A. Langari, Int. J. Quantum. Inform. 9, 1057 (2011).

[115] I. Titvinidze and G. I. Japaridze, Eur. Phys. J. B 32, 383 (2003). 